\title{
EL MOVIMIENTO EUGENÉSICO ESTADOUNIDENSE COMO CLAVE DEL ÉXITO DE LA EUGENESIA EN EL SIGLO XX Y LA POSIBILIDAD DE SU RETORNO EN EL SIGLO XXI
}

\author{
Jesús ParRa Sáez \\ Universidad de Murcia \\ bttp://dx.doi.org/10.15304/ag.37.2.4305
}

\section{Resumen}

Fundamentada en la milenaria idea del perfeccionamiento humano, la ideología eugenésica británica emergente a finales del siglo XIX tuvo en el movimiento eugenésico norteamericano el apoyo necesario para materializar una serie de políticas de carácter racial con el objetivo de perfeccionar la especie humana. El movimiento eugenésico estadounidense se constituyó como un paso clave en la extensión de una ideología que triunfó en numerosos Estados latinoamericanos y europeos, y que perdió todo su apoyo científico, político y social debido a su radicalización en Alemania durante el Holocausto nazi. En la actualidad, el debate en torno al pensamiento eugenésico y la posibilidad de su retorno ha crecido de forma exponencial con motivo del desarrollo biotecnológico, y de la posibilidad de una mejora humana biotecnológica que provoque graves daños al ser humano.

Palabras clave: política eugenésica, mejora humana biotecnológica, esterilización, degeneración racial, problemas éticos.

\begin{abstract}
Based on the millenarian idea of human enhancement, the British eugenics ideology emerging at the end of the 19th century had in the North American eugenics movement the necessary support to materialize a series of racial policies with the aim of perfecting the human species. The American eugenics movement was a key step in the extension of an ideology that triumphed in many Latin American and European states, and that it lost all its
\end{abstract}

Recibido: 20/09/2017. Aceptado: 20/12/2017. 
scientific, political and social support because of its radicalization in Germany during the Nazi Holocaust. Currently, the philosophical debate around eugenics thinking and the possibility of its return has grown exponentially due to biotechnological development, and the possibility of a human biotechnological enhancement that causes serious damage to human beings.

Keywords: eugenic politics, human biotechnological enhancement, sterilization, racial degeneration, ethical problems.

\section{Introducción}

La pretensión del ser humano por su auto-perfeccionamiento es tan antigua que impide fechar su origen de forma exacta. No obstante, desde la aparición del homo sapiens han sido varias las ocasiones en que el pensamiento eugenésico entendido como "búsqueda de la mejora de las cualidades humanas" se ha hecho público. Uno de los primeros vestigios de este pensamiento pueden rastrearse hasta el siglo $\mathrm{V}$ a. C., y más concretamente en el proyecto educacional espartano de la agogé a través del cual se pretendió conseguir toda una estirpe de seres humanos física-intelectualmente mejorados. Más tarde Platón en el Libro V de su obra La República, alentó el establecimiento de una serie de matrimonios pactados entre hombres y mujeres con las mejores capacidades físico-intelectuales con el fin de engendrar a los mejores ciudadanos posibles para su ciudad ideal. Posteriormente diversos movimientos socio-culturales - entre los que destacan el humanismo de los siglos XIV y XV o el movimiento ilustrado de siglo XVIII- serían relacionados con la ansiada mejora humana.

Sin embargo, esta milenaria pretensión comenzó a tomar forma - política e institucionalmente- gracias al apoyo de un entorno científico británico que, a finales del siglo XIX, se encontraba influido por la teoría darwiniana de la selección natural y por la propuesta de la «eugenesia» como herramienta para proteger al pueblo británico de la amenaza de la degeneración racial. Sin embargo, tras varios intentos fallidos de legalizar políticas eugenésicas como la esterilización, la ideología eugenésica no acabó de despegar en Reino Unido y fue al otro lado del Atlántico — concretamente Estados Unidos- donde tuvo lugar una explosión del pensamiento eugenésico cuyo resultado directo fue la creación de numerosas instituciones eugenésicas a lo largo de todo el continente americano y europeo, la implantación de leyes enfocadas a la protección de la raza, y en última instancia, gran parte de la estructura central de la ideología racial en virtud de la cual el nacionalsocialismo alemán acabó con la vida de millones de personas durante el Holocausto. A pesar de que tras el fin de la II Guerra Mundial el pensamiento 
eugenésico fue mundialmente rechazado, el movimiento estadounidense prosiguió su curso durante las siguientes tres décadas llevando a cabo un cambio terminológico en su discurso, usando términos relativos al cuidado de la salud y el bienestar de los ciudadanos y evitando expresar una preocupación abiertamente eugenésica ${ }^{1}$.

Actualmente, con motivo del desarrollo de nuevas técnicas biomédicas como los diagnósticos genéticos, la ingeniería genética, la investigación con células madre o la nanotecnología, ha surgido un amplio debate científicofilosófico entre los que legitiman su uso sobre el ser humano con fines perfeccionadores $^{2}$ (posthumanistas) y los que lo rechazan (bioconservadores) por motivos éticos. Un debate que al tiempo gira en torno a la posibilidad - poco plausible- de que tenga lugar el retorno de la ideología eugenésica, y con ella, el retorno de las políticas con carácter coercitivo en su nombre.

El objetivo del artículo es, por tanto, doble: 1) Mostrar cómo Estados Unidos se convirtió a lo largo del siglo XX —especialmente en su primer tercio y gracias a nombres como Harry Laughlin o Charles Davenport-, en el origen de toda una serie de ideales y políticas eugenésico-raciales representadas tanto por instituciones públicas como privadas que acabarían extendiéndose por numerosos Estados y que serían radicalizadas por parte del nacionalsocialismo alemán durante los años 30 y 40; y 2) Determinar la posibilidad de un retorno de la política eugenésica biológico-racial acontecida en el siglo XX con ocasión del actual desarrollo biotecnológico, a través del análisis ético-filosófico de los problemas que derivan de éste.

\section{La política británica y el inicio del movimiento eugenésico}

En sus inicios, la política eugenésica británica se encontraba influida por la idea de una posible degeneración racial de su población, supuestamente causada por el mestizaje y por la descendencia de individuos que presentaban ciertas deficiencias físicas, mentales y sociales. En este sentido, el gobierno británico siguió una política basada en la segregación de los individuos denominados "no aptos" entre los que se encontraban personas con problemas físicos, mentales y sociales. El punto álgido de su legisla-

\footnotetext{
${ }^{1}$ Un buen ejemplo es el representado por la denominada "lucha contra la pobreza" del presidente Lyndon B. Johnson.

${ }^{2}$ Por fines perfeccionadores se entiende de forma general la ampliación de caracteres o habilidades humanas positivas como la inteligencia, mientras que por fines terapéuticos se concibe la detección y eliminación de enfermedades.
} 
ción eugenésica tuvo lugar tras la finalización de la «Primera Conferencia Internacional de Eugenesia» de Londres en 1912 con la implantación de la conocida como «Mental Deficiency Act» (1913). Con esta ley el gobierno británico pretendía segregar a todos aquellos individuos que padecieran cualquier tipo de minusvalía mental a través de registros de enfermos y la prohibición matrimonial para quien padeciera alguna. Al tiempo, la legislación legitimaba la inclusión de individuos con deficiencias de carácter social o moral ${ }^{3}$, algo considerado por sus mayores críticos coetáneos como una violación de los derechos de los individuos que autorizaba "el encarcelamiento, supuestamente por demencia de personas a las que ningún médico se atrevería a declarar dementes" ${ }^{4}$. Durante los años 20 y 30 los integrantes del movimiento eugenésico británico, entre los que se encontraban importantes componentes del gobierno como Winston Churchill, crearon dos comités —el Comité Wood y el Comité Brock - con el fin de demostrar estadísticamente la tan temida degeneración de la raza británica y proponer la esterilización como herramienta eugenésica que solucionaría el problema.

A pesar de gozar de bastante éxito en el ámbito científico-médico como "la comunicación más acreditada sobre la esterilización que había aparecido hasta ese momento en cualquier país" ", los informes proporcionados por los comités al Ministerio de Salud no consiguieron el resultado esperado y la esterilización eugenésica jamás fue legalizada, debido entre otras cosas, a la fuerte oposición de la Iglesia Católica y a las noticias sobre el quehacer eugenésico-racial alemán en los últimos años de la II Guerra Mundial que asombraron al mundo.

\section{La emergencia de la política eugenésica norteamericana}

La política eugenésica estadounidense comenzó a principios del siglo XX de forma paralela a su homóloga británica. En 1905 el Estado de Indiana introdujo una medida eugenésica triple fundamentada sobre: 1) La prohibición del matrimonio para deficientes mentales y enfermos que padecieran alguna enfermedad transmisible; 2) La obligación de obtener un certificado sanitario por parte de antiguos pacientes y presidiarios; y 3) La anulación

${ }^{3}$ Capacidad económica nula, carencia de educación, o fácil caída en vicios como el alcohol y la prostitución.

${ }^{4}$ G. Chesterton, La eugenesia y otras desgracias, Sevilla, Espuela de Plata, 2012, p. 56.

${ }^{5}$ C. Blacker, "Voluntary Sterilization: The Last Sixty Years", The Eugenics Review 54/1 (1962), p. 16. 
de matrimonios declarados fraudulentos por haber tratado de eludir la legislación vigente.

Dos años más tarde se dio un paso fundamental en la carrera del movimiento eugenésico estadounidense que lo separaría por completo del estéril movimiento británico: la aprobación de la primera ley estatal de esterilización obligatoria. Esta ley, aprobada de nuevo en el Estado de Indiana, legitimaba la esterilización involuntaria de delincuentes habituales, locos e idiotas $^{6}$. En 1909, esta vez en el Estado de California, se aprobó una ley de esterilización dirigida a todo individuo clasificado como portador de deficiencias y residente en instituciones como asilos o prisiones, con el objetivo de mejorar las condiciones físicas, morales e intelectuales de la sociedad norteamericana.

Un año más tarde, se constituyó en Nueva York una de las instituciones eugenésicas más importantes de la historia del movimiento eugenésico mundial, y epicentro de la investigación en mejora humana de los Estados Unidos durante gran parte del siglo XX: la Eugenics Record Office. Su fundador, Charles Benedict Davenport ${ }^{7}$, es considerado por los expertos de la eugenesia como uno de sus principales teóricos. Esta institución realizó una enorme recopilación de datos ${ }^{8}$ biológico-hereditarios relativos, tanto a familias completas como a individuos solitarios procedentes de instituciones médicas públicas y privadas, como parte de un proyecto de análisis exhaustivo de los caracteres hereditarios de la especie.

En 1911 era fundada en Michigan una de las mayores instituciones eugenésicas estadounidenses, que relacionaba investigaciones relativas a la herencia de caracteres genéticos positivos y negativos con el estudio de medidas estrictamente preventivas como la higiene personal, la dieta o el ejercicio: la Race Betterment Foundation. Organizada por ésta, tuvo lugar en 1914 la «Primera Conferencia Nacional en la Mejora de la Raza», en la que se trató de mostrar a la sociedad norteamericana que la eugenesia se erigía como la única solución para la degeneración racial del pueblo estadounidense ${ }^{9}$.

${ }^{6}$ Más de 120 personas fueron esterilizadas en virtud de esta ley.

${ }^{7}$ Más allá de su participación en la Eugenics Record Office, Davenport dedicó gran parte de su vida profesional a defender - a través de conferencias en la Universidad de Harvard y obras como Heredity in Relation to Eugenics (1911) — la idea de que sin la ayuda del movimiento eugenésico el ser humano jamás podría progresar.

${ }^{8}$ Más de 65.000 informes de rasgos hereditarios y árboles genealógicos familiares completos para 1926.

${ }^{9}$ Esta afirmación es curiosamente similar a la que hizo el filósofo posthumanista Peter Sloterdijk en su ensayo Normas para el parque humano y su texto "El hombre operable" al 
Ese mismo año, el otro gran representante de la Eugenics Record Office, Harry Laughlin, creó un modelo de ley de esterilización muy exitoso que fue utilizado e impuesto en diversos territorios norteamericanos como Iowa y que, como veremos más tarde, sirvió de base fundamental de la primera ley eugenésica implantada por el gobierno nacionalsocialista alemán de Adolf Hitler. Esta ley modelo se caracterizaba por otorgar al Estado (cualquiera) la potestad de prohibir la procreación a personas "no aptas" por su biología hereditaria física y psicológicamente defectuosa. Finalmente, los individuos no aptos debían ser divididos en 10 tipos:

1) Débiles Mentales; 2) Insanos, (incluyendo el psicopático); 3) Criminalista (incluyendo el delincuente y rebelde); 4) Epiléptico; 5) Inebriado (incluyendo drogadictos habituales); 6) Enfermos (incluidos los tuberculosos, los sifilíticos, los leprosos y otros con enfermedades crónicas, infecciosas y segregables legalmente); 7) Ciegos (incluyendo aquellos con visión seriamente dañada); 8) Sordos (incluyendo aquellos con audiencia seriamente afectada); 9) Deformados (incluyendo lisiados); y 10) Dependientes (incluidos los huérfanos, los sin techo, los vagabundos y los indigentes). ${ }^{10}$

Con el inicio de una nueva década tuvo lugar en Nueva York la «Segunda Conferencia Internacional de Eugenesia» (1921), cuyos temas principales a debatir fueron el futuro de la raza humana y los problemas raciales relativos a la inmigración. Un año más tarde y siguiendo de forma clara la "ley modelo de Laughlin", se implantó en Iowa una ley de esterilización dirigida a los mismos 10 tipos de personas socialmente inadecuadas y de poca utilidad para la sociedad estadounidense que promulgaba aquélla. En 1923 se dio otro paso de gigante en el desarrollo del movimiento eugenésico con la constitución de la Sociedad Americana de Eugenesia, en la que participaban grandes pensadores como el científico e inventor Alexander Graham Bell o el economista Irving Fisher. A partir de este momento la preocupación por la degeneración racial y por ende, la lucha contra la inmigración fue una constante en la política estadounidense y los integrantes del movimiento eugenésico norteamericano se hacían eco del alarmante incremento de la inmigración y la ineficacia de las políticas eugenésicas anteriores para proteger la propia raza: "muchos seguidores estadounidenses de la eugenesia pensaron que las leyes de la restricción de matrimonios y los programas

señalar a las nuevas tecnologías como el único método útil para mejorar al ser humano ante el fracaso de los métodos tradicionales del humanismo (culturización y alfabetización) para separar a éste de su naturaleza más animal y de la barbarie que es capaz de mostrar.

10 Facing History and Ourselves National Foundation., Race and Membership in American History: The Eugenic Movement, Facing History and Ourselves, 2002, p. 192. 
de esterilización serían inútiles, si se consentía que perdurase la amenaza externa al vigor biológico nacional" ${ }^{11}$.

Frente a este sentimiento de terror nacional al inmigrante y a sus genes defectuosos, el gobierno norteamericano se apresuró a implantar una de las leyes más importantes en materia eugenésica de la historia de Estados Unidos: el «Acta de Inmigración» de 1924, que restringía la entrada de inmigrantes procedentes del sur de Europa por su condición socio-médica y la creencia generalizada de que las relaciones interraciales representaban "uniones disgénicas" en las que la raza superior —en este caso la blanca norteamericana-corría el riesgo de ver cómo su descendencia se contaminaría con los rasgos genéticos hereditarios defectuosos de razas inferiores. La ley gozó de gran popularidad, tanto en las altas esferas del país como entre los ciudadanos de a pie, y todavía más importante, fue vitoreada por parte del movimiento eugenésico mundial y adaptada por parte del gobierno alemán en los años 30 como solución al "problema inmigratorio".

En el mismo año que se estableció la ley de inmigración se impulsó una nueva ley de esterilización, esta vez en el Estado de Virginia. Esta ley permitía la esterilización involuntaria de enfermos mentales con patologías hereditarias en instalaciones estatales, como prisiones o instituciones médicas. En virtud de ella "el Estatuto de Virginia para la esterilización eugenésica dio un visto bueno legal a más de 8.300 operaciones" ${ }^{12}$, constituyéndose el conocido caso Buck v. Bell ${ }^{13}$ como uno de los procesos legales relativos al movimiento eugenésico más importantes, al ser la primera vez que la Corte Suprema de los Estados Unidos apoyaba la constitucionalidad de las prácticas eugenésicas esterilizadoras involuntarias, en virtud de la protección del bienestar de la población estadounidense. Para ese mismo año, al menos 26 estados norteamericanos habían aprobado leyes eugenésicas de esterilización.

${ }^{11}$ D. Kevles, La Eugenesia. ¿Ciencia o utopía? Una polémica que dura cien años, Barcelona, Planeta, 1986, p. 86.

12 P. Lombardo, "Involuntary Sterilization in Virginia: From Buck v. Bell to Poe v. Lynchburg”, Developments in Mental Health Law 3/3 (1983), p. 13.

${ }^{13}$ Véase: P. Lombardo, "Three generations, no imbeciles: new light on Buck v. Bell", New York University Law Review 60/30 (1985), pp. 30-62; y D. Betancor, "Leyes norteamericanas de esterilización eugenésica. Oliver Wendell Holmes y el caso Buck v.Bell”, Revista de la Facultad de Ciencias Jurídicas 7 (2002), pp. 30-45. 


\section{La política eugenésica de entreguerras y su maximización}

El período de entreguerras representa el éxito del movimiento eugenésico y su propagación a diversos países europeos y sudamericanos. Uno de los factores clave en la proclamación de este éxito fue el contexto social y económico de crisis que va desde el final de la Gran Guerra hasta la gran depresión de la economía norteamericana denominada "crack del 29", que acabó extendiéndose al sistema económico mundial. En este período de incertidumbre social y económica, la figura del inmigrante fue presentada como la de un enemigo de los derechos de los ciudadanos locales, que supuestamente acaparaba tanto los recursos de éstos como sus posibilidades de empleo:

Al público se le dio la clara impresión de que gran parte del conflicto estaba siendo agitado por organizadores inmigrantes que se habían infiltrado en la fuerza de trabajo estadounidense y que estaban trayendo caminos extraños y perjudiciales a una clase laboriosa básicamente pacífica. ${ }^{14}$

A partir de este momento y hasta finales de los años 70, la actuación eugenésica de la política estadounidense adquirió un sentido abiertamente racial. De forma bastante habitual, se repartió a los ciudadanos locales toda clase de publicidad de carácter racial con el fin último de desprestigiar a los inmigrantes, especialmente a los procedentes del país vecino, México. En este ambiente tuvo lugar en 1932 la «Tercera Conferencia Internacional de Eugenesia», la cual ayudó a expandir diversas ideas eugenésicas a numerosos estados extranjeros como Suecia o Alemania, que serían inmediatamente materializadas por los gobiernos de ambos países. Precisamente, el 1 de enero de 1934 el gobierno alemán implantó la conocida como «Ley sobre la prevención de la progenie con enfermedades hereditarias». Esta ley maximizaba los ideales eugenésicos defendidos en Norteamérica a través de la esterilización forzosa con el objetivo de evitar la degradación de la raza aria nórdico-germánica. ${ }^{15}$ Como muestra fehaciente de su importancia, sólo en 1934 se "ordenaron más de 56.000 esterilizaciones" ${ }^{16}$, mientras que para 1937 más de 225.000 personas habían sido esterilizadas. El número final de

${ }^{14}$ G. Allen, "The social and economic origins of genetic determinism: a case history of the American eugenics movement, 1900-1940 and its lessons for today", Genetica 99 (1997), p. 83.

${ }^{15}$ Lo más importante de esta ley es que introducía por primera vez en la historia un mandato de esterilización con validez nacional, algo que había sido ansiado en Estados Unidos sin éxito.

16 J. Barondess, "Care of the medical ethos: reflections on Social Darwinism, racial hygiene, and the Holocaust”, Annals of Internal Medicine 129/11 (1998), p. 893. 
esterilizados tras la caída del Tercer Reich en 1945 se cifra en más de medio millón de personas entre hombres y mujeres, muriendo más de 5.000 como consecuencia de las intervenciones.

En las páginas de la revista estadounidense especializada en eugenesia más importante del momento, la Eugenical News, se tradujo al inglés la ley alemana casi por completo como muestra de que los presupuestos eugenésicos norteamericanos —en especial su modelo de esterilización- habían triunfado más allá de sus fronteras y eran reconocidos por parte de las grandes potencias mundiales. Como consecuencia de la profunda inmersión de Alemania en el proyecto eugenésico la legislación norteamericana se intensificó, y un año más tarde de la implantación de la ley eugenésica alemana se aprobó en Oklahoma una nueva ley de esterilización involuntaria dirigida a criminales habituales. Una ley que hubiera pasado desapercibida entre el resto de normativas si no fuera por la polémica que suscitó el caso Skinner v. Estado de Oklahoma y la importancia de su fallo: los criterios para seleccionar los individuos que habían de ser esterilizados de forma involuntaria no eran coherentes. ${ }^{17}$ Gracias a este caso y a la reflexión del juez Douglas, gran parte del público general empezaba a cuestionarse si la política eugenésica norteamericana estaba actuando verdaderamente en función de la protección biológica y social de la raza o si se estaba haciendo en función del nivel socio-económico de los individuos. Además, el componente racial empezaba a ser cuestionado por lo que acontecía al otro lado del Atlántico y se anticipaba lo que sería la caída del movimiento eugenésico en Norteamérica tal y como se había desarrollado hasta el momento.

\section{El descubrimiento de los experimentos nazis y la reestructuración de la política eugenésica norteamericana tras la II Guerra Mundial}

Tras el establecimiento de las «Leyes de Núremberg» en 1935, en Alemania se pusieron en marcha todo tipo de proyectos eugenésicos que sirvieron de experimento antes de lo que sería la "Solución Final", esto es, el traslado indiscriminado de judíos y otros colectivos a los campos de concentración para su exterminio sistemático. En 1939 se iniciaron diversos programas experimentales en instituciones médicas como hospitales psiquiátricos, en los que se probaban distintas formas de eliminar a los deficientes mentales

${ }^{17}$ U.S. Supreme Court, “Skinner v. Oklahoma ex rel. Williamson” 316 U.S. 535 (1942), [En Línea] [Consultado el 10/02/17] Disponible en:

https://supreme.justia.com/cases/federal/us/316/535/case.html 
y físicos que presuntamente causaban tanto un gasto económico intolerable para la nación como un riesgo para la proliferación de la raza aria. Un año más tarde se puso en marcha el programa de "eutanasia forzada" conocido como «Aktion T4» ${ }^{18}$, y bajo el cual se crearon seis centros de eutanasia en los que se eliminaba a todo individuo considerado como improductivo o indeseable ${ }^{19}$ : "si la discapacidad privaba a una persona de una vida razonable, su aniquilación se llevaba a cabo en su propio bien y en el de la nación, para aliviar su propio sufrimiento y reducir la carga a la economía nacional" ${ }^{20}$. Los años que siguen al cese del programa Aktion T4 en 1941 hasta el final de la guerra en 1945, se caracterizaron por el genocidio judío y el descubrimiento mundial de los experimentos y acciones de los nazis.

En lo que a la mejora humana atañe, se crearon numerosos centros de experimentación eugenésica como el denominado "Comité del Reich para el Tratamiento Científico de las Enfermedades Severas Hereditarias y Congénitas» ${ }^{21}$, en los que se probaban nuevas formas de castración en hombres y de esterilización en mujeres, y se eliminaba a todo sujeto que mostrara síntomas evidentes de discapacidad. En otros centros se trataba de explotar el límite humano en referencia a sus capacidades positivas y no a la transmisión de caracteres negativos, comprobando la resistencia del ser humano en situaciones extremas, como su sumersión en aguas congeladas o la evolución de heridas quirúrgicas sin tratamiento médico. El balance final del Tercer Reich fue de más de 500.000 personas esterilizadas y más de 11 millones asesinadas.

A pesar de la intensa e íntima relación entre los eugenistas norteamericanos y alemanes, la intromisión de Estados Unidos en la Segunda Guerra Mundial en el año 1941 supuso un indudable rechazo de las políticas eugenésicas alemanas. Sin embargo, el movimiento estadounidense no acabó aquí, sino que sufrió una reestructuración, esencialmente terminológica, que permitió que siguiera su camino de forma mucho más cautelosa. Durante las décadas que siguen al Holocausto, los eugenistas norteamericanos evitaron utilizar el término eugenesia por su relación con el racismo y el nazismo, optando por establecer una nueva terminología eugenésica,

${ }^{18}$ En este programa se causó la muerte de 70.273 .

${ }^{19}$ Con el término "indeseable" se englobaba a diversos tipos de enfermedad mental y física como la esquizofrenia, la ceguera, la demencia, la epilepsia, y personas que padecieran de deformidades físicas o estuvieran paralíticos.

${ }^{20}$ S. Snyder, y D. Mitchell, "La eugenesia a ambos lados del Atlántico”, Intersticios. Revista Sociológica de Pensamiento Crítico 3/2 (2009), p. 61.

${ }^{21}$ En los dos años que este programa estuvo activo, más de 250.000 niños y adolescentes murieron. 
hablando de "control natal y planificación familiar". A pesar de todo, el ritmo de esterilizaciones prosiguió, y para el año 1945 más de 45.000 personas habían sido esterilizadas en Estados Unidos. Aunque es cierto que entre la década de los 50 y los 60 los investigadores en genética humana siguieron una línea de investigación alejada de los presupuestos eugenésico-raciales, éstos seguían su camino firme en el Estado de California al considerarse desde las altas esferas que era la mejor forma de preservar el bienestar social y la salud pública. Así, aún en la década de los 70 tuvieron lugar numerosas esterilizaciones en California con una motivación manifiestamente racial ${ }^{22}$ y de forma coercitiva, como muestra el famoso caso Madrigal $v$. Quilligan ${ }^{23}$ en el que diversas inmigrantes mexicanas fueron esterilizadas de forma involuntaria en un hospital estadounidense.

La legislación eugenésica de esterilización prosiguió en el Estado de California hasta el año 1979, cuando el legislador y presidente del Comité de Salud Art Torres, propuso la derogación de este tipo de leyes debido a la obsolescencia y ambigüedad de los criterios de selección de personas que debían ser sometidas a estas intervenciones, pues éstos ya no se correspondían con la medicina y salud pública modernas. La propuesta fue ampliamente apoyada desde distintas instituciones públicas y privadas de California, siendo finalmente aprobada en el Senado estatal. En los primeros años de la década de los 2000, el gobernador del Estado de California Gray Davis y el gobernador del Estado de Virginia Mark Warner, pidieron públicamente perdón a las personas que habían sido esterilizadas en virtud de la política eugenésica estatal, al tiempo que ofrecían una reclamación de sus derechos. Se evidencia el sentimiento de culpa que hay bajo este tipo de declaraciones y la importancia que tuvo el movimiento norteamericano en la historia de la eugenesia, por desarrollar ideas germinales que no se atrevieron a adoptar en otros estados como Reino Unido, por ser objeto de halagos de los ámbitos político y científico mundiales, y por ser capaz de seguir con su programa eugenésico llevando a cabo una restructuración de éste —especialmente terminológico y de cara al público— tras el horror del Holocausto.

${ }^{22}$ En la mayor parte de los casos, los sujetos intervenidos eran mujeres inmigrantes procedentes de México y Latinoamérica.

${ }^{23}$ Véase: A. Stern, "Esterilizadas en nombre de la salud pública: raza, inmigración y control reproductivo en California en el Siglo XX", Salud Colectiva 2/2 (2006), pp. 173190. 


\section{La influencia de la eugenesia norteamericana y su extensión mundial}

Como muestra fehaciente de la importancia del movimiento estadounidense en la extensión de la eugenesia, podemos encontrar dos ámbitos de desarrollo eugenésico que se iniciaron en Norteamérica y que fueron trasladados al otro lado del Atlántico: la creación de instituciones eugenésicas con el fin de realizar estudios biológico-raciales y promover el estudio de la eugenesia; y la implantación de políticas eugenésicas negativas en las que destacaban las leyes de esterilización involuntaria.

En cuanto al primer ámbito de desarrollo, a imagen y semejanza de la Eugenics Record Office y la Sociedad Americana de Eugenesia norteamericanas, se crearon toda una serie de instituciones eugenésicas desde Latinoamérica hasta Centroeuropa. En 1918 se crearon de forma paralela la Sociedad de Eugenesia de Sao Paulo y la Liga Pro-Saneamiento de Brasil24, con el objetivo de promover el estudio de la eugenesia y la preocupación acerca de la degeneración racial, tan extendida en el movimiento eugenésico norteamericano. En 1921 se creó en Argentina la denominada Liga Argentina de Profilaxis Social25, la cual ejerció — hasta la fundación en 1932 de la Asociación Argentina de Biotipología, Eugenesia y Medicina Social, y de la Sociedad Argentina de Eugenesia en 1945- de principal institución eugenésica del país que promovía el cuidado de la herencia humana y la prevención ante los problemas inmigratorios.

En 1922 se creó en Suecia el denominado Instituto Estatal de Biología Racial con el fin de distinguir antropológicamente al ciudadano sueco de cualquier otro, especialmente respecto a los denominados tattares. ${ }^{26}$ Con el mismo modus operandi del movimiento norteamericano, se realizó un amplio estudio higiénico-racial tanto de las características de los ciudadanos suecos como las de sus linajes, especialmente centrado en personas consideradas “asociales" o "incompetentes". En 1927 se fundó en Berlín el Instituto Kaiser Wilhelm de Antropología, Herencia Humana y Eugenesia,

${ }^{24}$ Véase: V. Souza et al, “The National Museum’s Physical Anthropology Archives: sources on the history of eugenics in Brazil", História Ciências Saúde-Manguinhos 16/3, 2009, pp. 763-777; y N. Stepan, The Hour of Eugenics. Race, Gender and Nation in Latin America, New York, Cornell University Press, 1991.

${ }^{25}$ Véase: G. Vallejo y M. Miranda, "Los Saberes del Poder: Eugenesia y Biopolítica en la Argentina del Siglo XX”, Revista de Indias 64/231 (2004), pp. 425-444.

${ }^{26}$ Etnia abiertamente marginada por los eugenistas debido a su presunta poca utilidad para el desarrollo de la sociedad sueca. Para más información véase: Spektorowski, "The eugenic temptation in socialism: Sweden, Germany, and the Soviet Union”, Society for Comparative Study of Society and History 46/1 (2004), pp. 84-106. 
cuya función principal consistía en dar legitimidad científica a las políticas raciales impuestas por el gobierno alemán, por lo que más tarde se convirtió en una de las instituciones eugenésicas más importantes del movimiento científico-político-racial del Tercer Reich. A finales del mismo año, el movimiento eugenésico norteamericano se había extendido a lo largo de todo el continente americano, teniendo lugar en La Habana la «Primera Conferencia Panamericana de Eugenesia y Homicultura» y en cuya resolución se propuso un "programa de eugenismo americano [de todo el continente] muy influido por el estadounidense". ${ }^{27}$

Posteriormente bajo la misma idea de degeneración racial y blanqueamiento de la población tan exitosa en Brasil, se fundó en México en 1931 la denominada Sociedad Mexicana de Eugenesia para el Mejoramiento de la Raza, constituida por profesionales de los ámbitos más diversos y con el objetivo último de afianzarse como representante principal del movimiento eugenésico mexicano ${ }^{28}$, y junto al movimiento argentino y uruguayo, como el principal movimiento eugenésico de Latinoamérica. Tan sólo tres años después se aprobaba en Uruguay el denominado "Código del Niño", el cual promovía que la reproducción estuviera guiada por los presupuestos eugenésicos básicos de unión entre individuos genético-hereditariamente sanos, de cara a la mejor salud y bienestar de los futuros nacidos. De forma paralela tenía lugar en Buenos Aires la «Segunda Conferencia Panamericana de Eugenesia y Homicultura», en la que se trató de promover el ideal eugenésico norteamericano de protección de la raza y la necesidad de que el Estado tomara cartas en el asunto de forma coercitiva si fuera necesario.

En lo que al segundo ámbito de desarrollo se refiere, de forma consecutiva se establecieron diversas leyes de esterilización en el territorio escandinavo. ${ }^{29}$ La primera ley de esterilización con rango nacional en Europa fue implantada en Dinamarca en 1929 y con carácter meramente voluntario para enfermos mentales recluidos. Sin embargo, 5 años más tarde la ley se convirtió en obligatoria para delincuentes y deficientes mentales, ascendiendo a 13.000 personas el número final de esterilizados hasta su derogación en 1967. Del mismo modo, en Noruega se implantaba en 1934 una ley de

${ }^{27}$ P. Barrán, "Biología, medicina y eugenesia en Uruguay”, Asclepio 51/2 (1999), p. 38.

${ }^{28}$ Véase: L. Suárez y López Guazo, "La influencia de la sociedad eugénica mexicana en la educación y en la medicina social”, Asclepio 51/2 (1999), pp. 51-84.

${ }^{29}$ Véase: A. Drouard, "Concerning Eugenics in Scandinavia. An Evaluation of Recent Research and Publications", Population. An English Selection 11 (1999), pp. 261-270; y P. Zylberman, "Eugénique à la scandinave: le débat des historiens", Medecine/Sciences 20/10 (2004), pp. 916-925. 
esterilización, enfocada a la esterilización voluntaria de individuos mentalmente enfermos que no podían hacerse cargo de sí mismos o de sus descendientes, como forma de evitar la degradación racial del pueblo noruego. En virtud de la ley fueron esterilizadas 44.000 personas antes de su derogación en 1977. Un año después de la implantación de la legislación noruega, en Suecia se establecía una ley de esterilización ${ }^{30}$ siguiendo el ejemplo de las leyes de esterilización involuntarias procedentes de Estados Unidos y Alemania, con el objetivo último de acabar con los deficientes mentales y con los individuos que eran considerados poco útiles para el desarrollo de la sociedad sueca y su bienestar. Bajo su mandato más de 62.000 personas serían esterilizadas en nombre de la eugenesia hasta su derogación en 1976.

\section{La posibilidad de una nueva eugenesia}

Desde los inicios del nuevo siglo, la amenaza de una nueva eugenesia está sonando con fuerza con motivo del desarrollo biotecnológico que partió en los años 70 con el desarrollo de la ingeniería genética y los diagnósticos genéticos, y que prosigue hoy con la nanotecnología y los implantes cibernéticos funcionales. En este paisaje tecnológico como telón de fondo, el debate filosófico-científico en torno a la eugenesia ha resurgido con más fuerza que nunca de mano de dos grandes corrientes de pensamiento habitualmente conocidas como bioconservadurismo y posthumanismo ${ }^{31}$. Así, la vieja pretensión de perfeccionar al ser humano ha tomado en la actualidad un rumbo nunca antes conocido, y es que tanto los científicos como los filósofos de pensamiento posthumanista proponen utilizar las más recientes técnicas biomédicas ${ }^{32}$ para mejorar cualitativamente las características físicas, psicológicas y moral-conductuales de nuestra especie. Es lo que se conoce generalmente como «nueva eugenesia» o «eugenesia liberal», aunque aquí la denominaré —en consonancia con la terminología habitualmente utilizada por los investigadores más representativos de ambas corrientes de pensamiento- «mejora humana biotecnológica» (Human Biotechnological Enhancement).

${ }^{30}$ Esta ley, inicialmente voluntaria, adquirió carácter obligatorio en 1941.

${ }^{31}$ En lo que atañe a la mejora humana biotecnológica, la corriente bioconservadora acepta la aplicación de las nuevas biotecnologías sobre el ser humano con objetivos exclusivamente terapéuticos (detección y eliminación de patologías), mientras que rechaza su aplicación con objetivos perfeccionadores (maximización de características positivas como la inteligencia o la resistencia física). Por el contrario, la corriente posthumanista legitima su aplicación en ambos casos. 
A pesar de que la mejora humana biotecnológica y el posible retorno de la eugenesia ha suscitado los más recientes debates filosóficos en el ámbito de la bioética y la ética aplicada, no hay consenso entre los investigadores a la hora de afirmar o rechazar el resurgimiento de la ideología eugenésica imperante en el siglo XX. De forma habitual, los pensadores más conservadores observan en las promesas de las nuevas biotecnologías -mejora radical de la salud, eliminación de enfermedades graves y maximización de características positivas como la inteligencia o la resistencia física- los mismos objetivos que ya defendía el movimiento eugenésico en los años 20 y 30. Algunos de los principales valedores de esta idea son la profesora de Sociología de la Salud y Bioética en la Universidad de Northampton Merryn Ekberg, el conocido historiador norteamericano Garland Allen, y el experto en mejora humana, inteligencia artificial y profesor en la Monash University Robert Sparrow. En palabras de este último: “muchas de las implicaciones de la nueva eugenesia son intervenciones genéticas que en sustancia — si no en la motivación — se parecen mucho a las defendidas por la vieja eugenesia". 33

En su artículo "The Old Eugenics and The New Genetics Compared", Ekberg entiende que, a pesar de las diferencias entre los rasgos distintivos del movimiento eugenésico en el siglo XX (pseudo-ciencia, política racial, discriminación femenina, discriminación hacia los discapacitados, colectivismo, y coerción), y las premisas de la mejora humana biotecnológica (ciencia, medicina preventiva, igualdad de oportunidades entre sexos, incremento de las posibilidades de los discapacitados, individualismo y voluntariedad), la ideología que hay tras ambas metodologías es la misma de hace un siglo. Como ejemplo paradigmático de esta situación, señala que la nueva genética sirve como herramienta opresora hacia los discapacitados, como lo hacía la vieja eugenesia ${ }^{34}$, y que, aunque una de las premisas de la nueva eugenesia es la individualidad, en última instancia la mejora de la especie humana es el objetivo final: "la nueva genética es un continuum de muchas preguntas sin respuesta planteadas por los antiguos eugenistas". ${ }^{35}$

\footnotetext{
${ }^{32}$ Manipulación genética, nanotecnología, selección embrionaria, clonación y cibernética.

${ }^{33}$ R. Sparrow, "A Not-So-New Eugenics: Harris and Savulescu on Human Enhancement", Hastings Center Report 41/1 (2011), p. 34.

${ }^{34}$ Los diagnósticos genéticos otorgan una amplia información genética del futuro nacido tanto a los profesionales médicos como a los padres, en base a la cual éstos podrían detener el embarazo de haberse detectado alguna patología que provoque una discapacidad determinada al neonato.

${ }^{35}$ M. Eckberg, "The Old Eugenics and the New Genetics compared", Social History of Medicine 20/3 (2007), p. 590.
} 
Por su parte, Allen vislumbra la existencia de una campaña desde los ámbitos científico, filosófico y periodístico para presentar las nuevas tecnologías como la solución perfecta a problemas biológicos, sociales y conductuales humanos, tal como ocurrió con la eugenesia en el seno del movimiento estadounidense. En base a esta idea, el pensador norteamericano afirma que el uso de técnicas biomédicas como la manipulación genética, con el objetivo de reparar o reemplazar genes defectuosos, se asimila en demasía a la pretensión eugenésica de eliminar caracteres hereditarios negativos que desembocó en la esterilización forzosa en nombre del bien común: “¿nos dirigimos hacia una nueva versión de la eugenesia? ¿Nos estamos dejando llevar por la falsa promesa de una solución tecnológica para los problemas que realmente se encuentran en la estructura de nuestra sociedad? Mi respuesta a estas preguntas es sí”. ${ }^{36}$

Por último, Sparrow deja entrever que las famosas pretensiones de igualdad y libertad de las nuevas herramientas tecnológicas darán como resultado la misma coerción y desigualdad características del movimiento eugenésico originario. Según la idea del pensador australiano, podría ocurrir que en una sociedad determinada se estableciera un ideal biológico, socio-cultural y comportamental de lo que sería un buen ciudadano -como el ideal ario nazi- para un desarrollo satisfactorio de aquélla. En tal caso, quienes no usen los medios biotecnológicos — sobre sí mismos o sobre los futuros nacidos- con tal objetivo estarían actuando en contra de su propia sociedad, es decir, estarían obrando de forma negligente. De este modo, si un sujeto se ve obligado de cualquier manera a someterse a una intervención con fines eugenésicos, la consecuencia directa es la coerción y no la libertad. ${ }^{37}$

Ahora bien, ¿están resurgiendo realmente los ideales del movimiento eugenésico? A diferencia de los pensadores más conservadores, entiendo que este nuevo movimiento al que denominan indistintamente «nueva eugenesia», «eugenesia liberal» o «eugenesia de mercado», es una corriente que trata de lograr una mejora cualitativa de las características humanas - acabar con la enfermedad, retrasar el envejecimiento, acabar con la violencia y el racismo, o hacernos más inteligentes y morales-, de forma no coercitiva y sin adhesión a ningún tipo de ideología o estamento político. Precisamente

${ }^{36}$ G. Allen, "Is a new eugenics afoot?", Science 294 (2001), p. 59.

${ }^{37}$ Frente a la libertad de los padres para elegir los rasgos genéticos de sus futuros hijos, emerge la obligación de escoger una combinación genética determinada que permita al futuro nacido estar en igualdad de condiciones con el resto de miembros pertenecientes a su sociedad. 
lo que distingue al movimiento eugenésico de lo que he preferido denominar «mejora humana biotecnológica» es su metodología. Anteriormente pudimos observar cómo las prácticas eugenésicas se reducían a uniones pactadas y dirigidas entre personas con características físico-intelectuales óptimas, la prohibición de reproducirse para los “menos aptos", la esterilización y la eliminación. Todo ello establecido de forma coercitiva por parte de gobiernos tanto liberales como autoritarios. ${ }^{38}$ Sin embargo, la metodología de la mejora humana biotecnológica es abiertamente distinta a la de aquélla. Cuando el filósofo neozelandés Nicholas Agar acuñó el término eugenesia liberal en 1998 lo hizo con base en esta diferencia metodológica e ideológica:

Mientras que la postura clásica de la eugenesia autoritaria buscaba producir ciudadanos que se ajustaran a un sólo modelo, la marca distintiva de la nueva eugenesia liberal es la neutralidad del Estado (...) La eugenesia liberal propone que lleguemos a los mejores arreglos internos y sociales permitiendo a los futuros padres guiarse por sus valores al momento de elegir acrecentamientos. ${ }^{39}$

Lo mismo opina el profesor de derecho en la University of San Diego y director del "Centro de Políticas de Derecho Sanitario y Bioética» (Center for Health Law Policy and Bioethics) Dov Fox, quien entiende que la eugenesia sufre una indudable transformación tanto en su concepción ideológica como en sus medios, fundamentada sobre el control de la ideología eugenésica por parte de la genética, la neutralidad gubernamental y la libertad parental que desemboca en lo que también él denomina «eugenesia liberal ${ }^{40}$ y que tiene 3 pilares principales: 1) la voluntariedad de someterse a intervenciones de mejora, 2) la individualidad en la toma de decisiones, en tanto que la mejora está enfocada a individuos concretos y no a grupos, y 3) la neutralidad estatal, que evita la emisión de medidas eugenésicas obligatorias.

No obstante y aunque la mejora humana biotecnológica se erige como un movimiento ideológica y metodológicamente distinto al movimiento eugenésico, es evidente que guarda en sí toda una serie de problemas de índole ético-moral que, en casi todos los casos, está relacionada con la ambigüe-

\footnotetext{
${ }^{38}$ Se ha entendido en numerosas ocasiones de forma errónea que las políticas eugenésicas características del siglo XX tienen como ejecutor común un gobierno de carácter totalitario que usa la coacción personal como forma de persuasión. Por eso es importante tener en cuenta que el movimiento eugenésico en un país democrático-liberal como Estados Unidos fue clave en el desarrollo y éxito de la eugenesia.

${ }^{39}$ N. Agar, "Liberal Eugenics”, Public Affairs Quarterly 12/2 (1998), pp. 137-139.

${ }^{40}$ Véase: D. Fox, “The Illiberality of liberal Eugenics”, Ratio 20/1 (2007), pp. 1-25.
} 
dad de las consecuencias de la aplicación de ciertas herramientas biotecnológicas sobre aspectos fundamentales del ser humano como es la construcción de su identidad personal. Como afirma la filósofa española Adela Cortina: "las investigaciones se encuentran todavía en los comienzos y sería muy difícil indicar cómo practicarlas y con qué consecuencias al medio y largo plazo". ${ }^{41}$ Precisamente, esta ambigüedad, o más bien, el conjunto de posibles consecuencias éticas teóricas y prácticas del perfeccionamiento humano biotecnológico, constituye actualmente el asunto principal a dilucidar por los teóricos de la nueva eugenesia. Así, no es casualidad que gran parte de las investigaciones de los centros de investigación en ética práctica o aplicada más importantes del momento no se preocupen tanto de si la human biotechnological enhancement sigue los preceptos de la vieja eugenesia, sino de lo lejos que pueden hacernos llegar las nuevas tecnologías y en qué medida una mejora de las magnitudes pretendidas pueda afectar a factores inherentes a la humanidad, como la identidad y dignidad humanas, o las desigualdades biológicas, sociales y económicas que pueda provocar. En suma, se está tratando de establecer la gama de beneficios y riesgos que las nuevas tecnologías biomédicas pueden ofrecer y en qué medida podemos legitimar o no su uso para objetivos no exclusivamente terapéuticos.

\section{Los problemas éticos del perfeccionamiento humano biotecnológico}

El debate contemporáneo respecto a la mejora humana, más allá de intentar dilucidar el posible retorno de los viejos ideales eugenésicos, abarca toda una serie de problemas éticos y sociales asociados a las nuevas tecnologías de mejora que provocan el rechazo de su uso con objetivos noterapéuticos por parte de las corrientes de pensamiento más conservadoras. Aquí mencionaré tres: 1) afección a la dignidad humana, 2) afección a la identidad humana, y 3) desigualdad biológica, social y económica.

Diversos pensadores de renombre como Leon Kass, George Annas o Jürgen Habermas vaticinan problemas relativos al reconocimiento que los sujetos humanos hacen del resto (otredad) en cuanto tal (su valor), esto es, problemas en la dignidad humana ${ }^{42}$. Éste último en su obra El futuro

${ }^{41}$ A. Cortina, “¿Es la biomejora moral un imperativo ético?”, Sistema 230 (2013), p. 13.

42 De forma general se entiende por dignidad humana el reconocimiento del valor que hay en cada ser humano como perteneciente a la especie humana, que establece una determinada igualdad entre todos sus componentes con independencia de las circunstancias biológicas y sociales que los envuelven, y que les otorga el estatus de "fin en sí mismo" en el 
de la naturaleza humana, ¿̇hacia una eugenesia liberal? y como máximo representante de la corriente filosófica más conservadora, afirma que en la medida en que la dignidad humana está "ligada a la simetría de las relaciones" 43 , ha de protegerse el reconocimiento recíproco que se hacen los seres humanos entre sí en el seno de sus relaciones como miembros de la misma especie: De este modo, intervenciones biotecnológicas que rompan este reconocimiento recíproco — por ejemplo, con la mejora radical de diversos individuos mientras que otros permanecen "obsoletos"- suponen un grave problema para el futuro de la humanidad. Esta preocupación no sólo ocupa las investigaciones filosóficas, sino que instancias gubernamentales como el «Consejo Presidencial de Bioética» de los Estados Unidos ha realizado informes en torno a la dignidad humana, su relación con el respeto por el otro y su autonomía, y la amenaza que suponen las nuevas tecnologías para ambas $^{44}$. En dichos informes, filósofos pertenecientes a ambas corrientes de pensamiento - como Kass o Bostrom- han tomado la palabra para aportar su punto de vista. El primero aludiendo a la pérdida de humanidad inherente al sometimiento sin peros a las tecnologías de mejora, ${ }^{45}$ que es ofrecido a día de hoy por los detractores de la validez del concepto de dignidad humana en los debates éticos. El segundo por su parte, afirmando una gradualidad en la dignidad en tanto que cualidad humana que ha de ser promovida, respetada y cultivada, que puede ser mejorada a través de medios biotecnológicos para que, entre otras cosas, los que no gocen del reconocimiento de dicha cualidad por parte de los demás, puedan hacerlo en el futuro.

Por otro lado, los detractores de la mejora humana biotecnológica en su vertiente perfeccionadora —especialmente Andorno y Habermas - aluden de igual modo a la amenaza que supone ésta para aquello que nos hace únicos a cada uno de los seres humanos: la identidad personal. ${ }^{46} \mathrm{La}$ idea

sentido kantiano y lo exime de ser utilizado como un mero objeto. También es considerada en ocasiones como aquello estimable y valioso en el ser humano.

${ }^{43}$ J. Habermas, El futuro de la naturaleza humana. ¿Hacia una engenesia liberal?, Barcelona, Paidós, 2010, p. 50.

${ }^{44}$ Véase: President's Council on Bioethics, Beyond Therapy Biotechnology and the Pursuit of Happiness, Washington, 2003; y President's Council on Bioethics, Human Dignity and Bioethics, Washington, 2008.

${ }^{45}$ Se refiere a cuestiones relativas a la interrelación entre la dignidad humana y aquellas acciones que constituyen una amenaza para ella, como la creación de seres idénticos (clonación), la cosificación de embriones humanos en la experimentación o la implementación de tecnología cibernética en la vida diaria (chips, implantes cocleares, órganos artificiales).

${ }^{46}$ Con identidad se alude aquí al conjunto de todos aquellos (genética, experiencias, relaciones, actos, educación, deseos, preferencias...) que hace que un ser humano se 
es que un sujeto que sufre una o varias intervenciones perfeccionadoras sufrirá una crisis de identidad tras aquéllas al constituirse como un sujeto con características distintas, con nuevos deseos y preferencias diferentes. Uno de los principales valedores de esta teoría es el filósofo neozelandés Nicholas Agar, quien en su obra Truly Human Enhancement afirma que este tipo de intervenciones podría afectar gravemente a lo que denomina «memoria autobiográfica» ${ }^{47}$, esto es, el totum que conforma la identidad de un sujeto - experiencias, recuerdos, deseos, responsabilidades- a lo largo de su vida. Un sujeto mejorado de forma radical tendrá, pues, una serie de características que no se corresponderían con este totum que tenía en el pasado. En suma, el sujeto post-intervención no se identificaría con el sujeto pre-intervención y no tendría más remedio que enfocar su vida hacia el futuro, forjando una nueva identidad. Sin embargo, pensadores con posturas cercanas a la corriente posthumanista como el profesor de filosofía moral en la George Washington University David DeGrazia, así como otros adscritos al movimiento bioconservador como el filósofo-político y profesor en la Universidad de Harvard Michael Sande ${ }^{48}$ no creen que este reclamo esté bien fundamentado, pues los que atisban una futura afección a la identidad humana de manos de las tecnologías de mejora no acaban de delimitar o definir el concepto de identidad y en qué modo se vería afectado por éstas. Otros como Bostrom y $\mathrm{Ord}^{49}$, niegan de forma flagrante que vaya a tener lugar tal crisis de identidad, sino que, más bien, tendrá lugar un desarrollo del individuo y su personalidad o identidad análogo al que todo ser humano tiene en el trayecto vital que va desde la infancia hasta la adultez.

Por último, instituciones como la Fundación Nacional de Ciencias de los Estados Unidos (U.S. National Science Foundation) -también conocida como NSF - han llevado a cabo investigaciones con el objetivo de emitir informes exhaustivos en torno a la situación ética derivada del uso biotecnológico sobre el ser humano con fines perfeccionadores. En 2009 la fundación publicó uno de estos informes bajo el nombre de "Ethics of $\mathrm{Hu}$ -

constituya como un ser único y distinto entre el resto, y que influye también en su forma de ser y actuar. En otros contextos también se le denomina "yo", "personalidad" e incluso "carácter" o "alma".

${ }^{47}$ N. Agar, Truly human enhancement: a philosophical defense of limits, MIT Press, 2014, p. 60.

${ }^{48}$ Véase: D. DeGrazia, "Enhancement Technologies and Human Identity", Journal of Medicine and Philosophy 30, pp. 261-283; y M. Sandel, Contra la perfección. La ética en la era de la ingeniería genética, Barcelona, Marbot Ediciones, 2015.

${ }^{49}$ Véase: N. Bostrom, y T. Ord, “The reversal test: eliminating Status Quo bias in applied ethics”, Ethics 116 (2006), pp. 656-679. 
man Enhancement: 25 Questions and Answers" 50, en el que respondían de forma concisa cuestiones éticas fundamentales de la mejora humana biotecnológica. Uno de los problemas más abordados en este tipo de informes, y al tiempo más preocupante, es el de las desigualdades biológicas, sociales y económicas provocadas por las tecnologías de mejora. Como avisa el informe: "las ventajas adquiridas por las personas mejoradas también implican una desventaja relativa para los no mejorados, ya sea en deportes, oportunidades de empleo, rendimiento académico o cualquier otra área". ${ }^{51}$ Esta es también una de las principales preocupaciones para filósofos morales y del derecho como el australiano Robert Sparrow y el norteamericano Francis Fukuyama. En una de sus obras predilectas - El fin del hombre - ${ }^{52}$ Fukuyama pone su foco de atención sobre las consecuencias políticas, sociales y existenciales para el futuro de la humanidad como consecuencia de la pretendida mejora humana perfeccionadora. Una de las consecuencias más significativas - ya aludida por Robert Malthus en su Ensayo sobre la población - sería la relativa a una presunta prolongación vital radical del ser humano, la cual entraría en conflicto con los recursos limitados del planeta Tierra y la posibilidad de satisfacer las necesidades de una especie que no para de crecer, y que vive progresivamente una vida más larga (crecimiento de la población y envejecimiento de la sociedad). En consonancia con esta idea y de forma muy reciente, expertos en matemática y estadística como el profesor en la Chalmers University of Technology Olle Häggström ${ }^{53}$ han mostrado su preocupación al respecto desde el punto de vista de la probabilidad y la estadística.

Teniendo en mente el avance biotecnológico y como medida preventiva para evitar su uso con fines perfeccionadores que ocasione problemas fundamentales para el ser humano, estas preocupaciones de carácter interdisciplinar también han sido recogidas, tanto por parte de la legislación internacional, como por distintas legislaciones nacionales. A nivel internacional la primera instancia reguladora es la denominada Declaración Universal

${ }^{50}$ Véase: F et al. Allhoff, "Ethics of Human Enhancement: 25 Questions \& Answers", US National Science Foundation, 2009. Disponible en: http://www.humanenhance.com/ NSF_report.pdf

${ }^{51} \mathrm{~F}$ et al. Allhoff, "Ethics of Human Enhancement: 25 Questions \& Answers", US National Science Foundation, 2009, p. 21.

${ }^{52}$ Véase: F. Fukuyama, El fin del hombre: consecuencias de la revolución tecnológica, Barcelona, Ediciones B, 2002.

${ }^{53}$ O. Häggström, Aquí hay dragones. Ciencia, tecnología y el futuro de la humanidad, TEELL, 2016. 
de los Derechos Humanos aprobada por la ONU en 1948, que establece una serie de derechos intrínsecos de todos los seres humanos con carácter universal e inviolable. Posteriormente, y con motivo del desarrollo de nuevas técnicas biomédicas como la ingeniería genética, se aprobó en la XXIX Conferencia General de la UNESCO de 1997 la Declaración Universal sobre el Genoma Humano y los Derechos Humanos, en la que se proclamó que ningún tipo de avance científico puede prevalecer sobre los derechos y libertades de los seres humanos bajo ningún concepto.

Por su parte, en lo que respecta a legislaciones de carácter nacional como la española, nuestra Constitución protege a través de su artículo 10 la dignidad humana y el libre desarrollo de la personalidad como derecho inviolable de la humanidad, al tiempo que protege la integridad moral del sujeto humano con el artículo 15. Del mismo modo, a través de las leyes 35/1988 y 42/1988 se estableció el control de técnicas de reproducción asistida y el uso de embriones, fetos, células y tejidos de origen humano. En la Ley orgánica 10/1995 se establecen penas relativas a delitos de manipulación genética (especialmente en cuanto a su uso no terapéutico). Posteriormente, entre el año 2003 y 2007 se promulgaron nuevas leyes en torno a las tecnologías biomédicas, como la ley 7/2003 para la regulación de pre-embriones humanos no viables para la fecundación in Vitro, la ley 45/2003 como reforma de la anterior ley 35/1988, la ley 14/2006 sobre técnicas de reproducción humana asistida o la ley 14/2007 sobre investigación biomédica. Así, las distintas legislaciones que van desde un nivel de seguimiento nacional (tanto en España como en la mayor parte de países europeos y americanos) hasta un nivel internacional establecen que los nuevos medios biotecnológicos únicamente pueden ser aplicados sobre seres humanos con objetivos manifiestamente terapéuticos.

\section{Conclusiones}

En lo que toca al primer objetivo del artículo, la fundación y desarrollo de poderosas instituciones eugenésicas en Norteamérica, como por ejemplo la Eugenics Record Office, la Race Betterment Foundation, o la Sociedad Americana de Eugenesia dio lugar a la aparición de toda una serie de instituciones análogas en numerosos países de Latinoamérica y Europa. Al tiempo, el movimiento eugenésico norteamericano fue clave para la instauración de una política eugenésica que materializó los ideales genéticohereditarios propuestos por los primeros defensores de la eugenesia en el 
siglo XIX. Estados Unidos fue el primer país en implantar leyes relativas a la eugenesia negativa, como lo fue la ley de esterilización de 1907 del Estado de Indiana y de los últimos en derogarlas (finales de los años 70). De esta manera, tanto la ley de inmigración de 1924, como la ley modelo de esterilización propuesta por Laughlin 10 años antes, sirvieron de precedente para el establecimiento en la Alemania nazi de la «Ley sobre la prevención de la progenie con enfermedades hereditarias» de 1934 y las leyes con carácter eugenésico-racial incluidas en las Leyes de Núremberg en 1935.

Más allá de la constante relación eugenésica entre Estados Unidos y Alemania, la legislación norteamericana fue motivo de imitación para otros movimientos europeos, y se vio traducida en importantes políticas eugenésicas negativas que comenzaron con la implantación de la ley de esterilización danesa en 1929 — veintidós años más tarde de la primera ley norteamericana de este tipo-, siguieron con la implantación gradual de leyes paralelas en países como Noruega o Suecia, cuyas víctimas se cuentan por cientos de miles, y que no fueron derogadas hasta la década de los 70 al igual que en Norteamérica. Finalmente, el descubrimiento del quehacer eugenésico alemán durante la Segunda Guerra Mundial, dejó en un segundo plano la importancia del movimiento eugenésico norteamericano en el desarrollo de las ideas eugenésicas originarias que habían permanecido en estado germinal hasta entonces. No obstante, desde el movimiento estadounidense se supo superar el enorme bache que suponía para sus objetivos el rechazo mundial de las acciones eugenésicas alemanas, llevando a cabo una reforma terminológica e ideológica de sus presupuestos que permitió que la política eugenésica norteamericana siguiera su curso dos décadas más.

Por otro lado y en lo que se refiere al segundo objetivo del artículo, esto es, el resurgimiento del movimiento eugenésico y el establecimiento de políticas con motivo del desarrollo de nuevas tecnologías biomédicas, la condena mundial de los actos del nazismo que tan relacionados estuvieron con la eugenesia - cuya consecuencia principal fue el establecimiento de una serie de derechos inviolables del ser humano con validez internacional en la Declaración Universal de los Derechos Humanos-; la pretensión cada más extendida de crear sociedades fundamentadas en la libertad y la pluralidad; las legislaciones nacionales que actualmente protegen - junto al derecho internacional- aspectos esenciales del ser humano como la dignidad, la libertad y el genoma humanos, hace prácticamente inviable el retorno de la ideología eugenésica en términos propios al movimiento eugenésico de los siglos XIX y XX. No obstante, a medida que las nuevas tecnologías biomédicas se desarrollan los diversos consejos o comités de bioética 
nacionales - el norteamericano es un ejemplo-, comunitarios - como los relativos a la Comunidad Europea-, e internacionales — como los propios a la UNESCO_ tratan de establecer los posibles problemas éticos de su uso sobre seres humanos de cara a su aceptación o rechazo legal.

Al mismo tiempo, lo que en el debate contemporáneo se conoce como «nueva eugenesia», «eugenesia liberal» o, quizás mejor, «mejora humana biotecnológica», está construida sobre una serie de principios fundamentados en la libertad, autonomía, e individualidad de los ciudadanos que se presentan como esencialmente distintos a los del movimiento eugenésico originario, a saber, la coerción, la colectividad, la estatalización y el racismo. Precisamente esta diferencia en sus supuestos fundamentales separa ideológica y metodológicamente ambos movimientos, y aleja la posibilidad de que la eugenesia liberal sea confundida o identificada con el viejo movimiento eugenésico. Sin embargo, la mejora humana biotecnológica tal y como ha sido propuesta desde los sectores más progresistas — especialmente la indistinción entre intervención biomédica terapéutica y perfeccionadora que hacen los pensadores posthumanistas-, presenta toda una serie de problemas de índole ético-moral relativos a su alcance y consecuencias para el futuro de la humanidad, que requieren una respuesta crítica que, probablemente, correrá a cargo de la filosofía y las éticas aplicadas tal y como muestran los últimos estudios de dos centros de investigación ética de renombre: el Oxford Uehiro Centre for Practical Ethics ${ }^{54}$, que actualmente está centrando esfuerzos en determinar la posibilidad de llevar a cabo una mejora de la estructura moral o conductual humana —eliminación de conductas negativas como el racismo, la violencia o la xenofobia y potenciación de conductas positivas como el altruismo y la empatía- a través de la alteración artificial del cerebro; y el Centre for Research Ethics and Bioethics ${ }^{55}$ de la Universidad de Uppsala, que en relación directa con la investigación del Uehiro Centre, trata de establecer desde su línea de investigación de neuroética los aspectos prácticos éticos y legales de la alteración de biología cerebral humana con motivo de la investigación científica y la aparición de nuevos tratamientos y aplicaciones clínicas.

\footnotetext{
${ }^{54}$ Véase: https://www.practicalethics.ox.ac.uk/article/research

${ }^{55}$ Véase: http://www.crb.uu.se/research/
} 


\section{Bibliografía}

Agar, N., "Liberal Eugenics", Public Affairs Quarterly 12/2 (1998), pp. 137-155.

Agar, N., Truly human enhancement: a philosophical defense of limits, MIT Press, 2014.

https://doi.org/10.7551/mitpress/9780262026635.001.0001

Allen, G., "The social and economic origins of genetic determinism: a case history of the American eugenics movement, 1900-1940 and its lessons for today”, Genetica 99 (1997), pp. 77-88.

https://doi.org/10.1007/BF02259511 https://doi.org/10.1023/A:1018396529332

Allen, G., "Is a new eugenics afoot?", Science 294 (2001), pp. 59-61. https://doi.org/10.1126/science.1066325

Allhoff, F et al., "Ethics of Human Enhancement: 25 Questions \& Answers", US National Science Foundation, 2009.

Barondess, J., "Care of the medical ethos: reflections on Social Darwinism, racial hygiene, and the Holocaust", Annals of Internal Medicine 129/11 (1998), pp. 891-898.

https://doi.org/10.7326/0003-4819-129-11_Part_1-199812010-00010

Barrán, P., "Biología, medicina y eugenesia en Uruguay”, Asclepio 51/2 (1999), pp. 11-50.

https://doi.org/10.3989/asclepio.1999.v51.i2.309

Blacker, C., "Voluntary Sterilization: The Last Sixty Years", The Eugenics Review 54/1 (1962), pp. 9-23.

Chesterton, G., La engenesia y otras desgracias, Sevilla, Espuela de Plata, 2012.

Cortina, A., “¿Es la biomejora moral un imperativo ético?”, Sistema 230 (2013), pp. 3-14.

Eckberg, M., "The Old Eugenics and the New Genetics compared", Social History of Medicine 20/3 (2007), pp. 581-593. https://doi.org/10.1093/shm/hkm075

Facing History and Ourselves National Foundation., Race and Membership in American History: The Eugenic Movement, Facing History and Ourselves, 2002.

Habermas, Jürgen., El futuro de la naturaleza humana. ¿Hacia una eugenesia liberal?, Barcelona, Paidós, 2010.

Kevles, D., La Eugenesia. ¿Ciencia o utopía? Una polémica que dura cien años, Barcelona, Planeta, 1986. 
Lombardo, P., "Involuntary Sterilization in Virginia: From Buck v. Bell to Poe v. Lynchburg", Developments in Mental Health Law 3/3 (1983), pp. 13-14.

Snyder, S y Mitchell, D., "La eugenesia a ambos lados del Atlántico", Intersticios. Revista Sociológica de Pensamiento Crítico 3/2 (2009), pp. 49-65.

Sparrow, R., "A Not-So-New Eugenics: Harris and Savulescu on Human Enhancement", Hastings Center Report 41/1 (2011), pp. 32-42. https://doi.org/10.1002/j.1552-146X.2011.tb00098.x 